\title{
Locomotion Mode Recognition with Inertial Signals for Hip Joint Exoskeleton
}

\author{
Gang Du, ${ }^{1}$ Jinchen Zeng $\mathbb{D}^{2},{ }^{2}$ Cheng Gong, ${ }^{3}$ and Enhao Zheng $\mathbb{D}^{4}$ \\ ${ }^{1}$ School of Information Engineering, China University of Geosciences, Beijing 100083, China \\ ${ }^{2}$ Faculty of Electrical Engineering, Mathematics and Computer Science, Technische Universiteit Delft, Delft 2600AA, Netherlands \\ ${ }^{3}$ College of Engineering, Peking University, Beijing 100871, China \\ ${ }^{4}$ The State Key Laboratory of Management and Control for Complex Systems, Institute of Automation, Chinese Academy of Sciences, \\ Beijing 100190, China
}

Correspondence should be addressed to Jinchen Zeng; j.zeng-4@student.tudelft.nl and Enhao Zheng; enhao.zheng@ia.ac.cn

Received 18 November 2020; Revised 15 April 2021; Accepted 11 May 2021; Published 24 May 2021

Academic Editor: Musa L. Audu

Copyright (C) 2021 Gang Du et al. This is an open access article distributed under the Creative Commons Attribution License, which permits unrestricted use, distribution, and reproduction in any medium, provided the original work is properly cited.

\begin{abstract}
Recognizing locomotion modes is a crucial step in controlling lower-limb exoskeletons/orthoses. Our study proposed a fuzzy-logic-based locomotion mode/transition recognition approach that uses the onrobot inertial sensors for a hip joint exoskeleton (active pelvic orthosis). The method outputs the recognition decisions at each extreme point of the hip joint angles purely relying on the integrated inertial sensors. Compared with the related studies, our approach enables calibrations and recognition without additional sensors on the feet. We validated the method by measuring four locomotion modes and eight locomotion transitions on three able-bodied subjects wearing an active pelvic orthosis (APO). The average recognition accuracy was $92.46 \%$ for intrasubject crossvalidation and $93.16 \%$ for intersubject crossvalidation. The average time delay during the transitions was $1897.9 \mathrm{~ms}(28.95 \%$ one gait cycle). The results were at the same level as the related studies. On the other side, the study is limited in the small sample size of the subjects, and the results are preliminary. Future efforts will be paid on more extensive evaluations in practical applications.
\end{abstract}

\section{Introduction}

Lower-limb exoskeletons/orthoses serve as important roles in rehabilitation, industrial manufacture, and other humancentered areas [1]. The specifically designed mechanical structures and the control strategies can alleviate the loads on the human body and thus increase the wearer's absolute strength in heavy load bearing or endurance in long-term tasks. There are various types of exoskeletons according to the active joints, such as whole-body exoskeletons (e.g., BLEEX [2] and HAL [3]) and single-joint ones [4] (e.g., hip joint and ankle joint). The hip joint connects the lower extremity and the trunk. The hip joint's primary function is to support the weight of the body in both static (e.g., standing) and dynamic (e.g., walking) postures [5]. The development of the hip joint exoskeleton is a hot research topic in this area. There are many groups developing hip joint exoskeletons (or active pelvic orthoses) all over the world
[6-13]. The assistance on the hip joint helps to stabilize the locomotion [6-9], optimize the metabolic cost $[10,11]$, adjust the abnormal gait patterns [12], and reduce the extra loads on the spine [13], according to the design of the exoskeleton.

One primary step in exoskeleton control is to recognize lower-limb motion intents accurately. It bridges the gap between the human sensorimotor system and the external robotic controllers, the performance of which determines the safety and working efficiency of the whole system [4]. The recognition tasks include gait phase estimation/detection, locomotion mode recognition, and other joint motion parameter estimations. Locomotion mode recognition involves the ambulation modes on different terrains (e.g., level ground and stairs) and the nongait patterns (e.g., standing). The recognition system should recognize the current modes and mode transitions accurately on multiple subjects. The recognition approach comprises the sensing system and the 
processing algorithms. The processing algorithms are usually designed based on the signal features of the sensing system. Previous studies on this area suggest that the neuralmechanical signal fusion method can produce satisfactory recognition results (e.g., accuracies and time latency). The neural signals are usually measured from the muscle signals (e.g., electric activities represented as surface EMG or shape changes represented by the noncontact capacitive sensors). The mechanical signals are measured from the inertial measurement units (IMUs) and loadcell sensors. The sensor nodes can be integrated into the mechanical structure of the exoskeletons. The muscle signals respond faster than the mechanical signals. However, they convey more noises, and the mechanical signals can produce signals with high repeatability due to the advancement of sensing technology. The combination of the signals can compensate each other to get better performance.

The target for locomotion mode recognition is to produce an accuracy as high as possible with the least intervention on the human body. The muscle signals require additional electrodes or front-ends on the human body, which decreases the convenience and the potential willingness of uses. Another limitation is that the recognition parameters should be calibrated for each individual, increasing the time needed before use. For hip joint exoskeleton control, many researchers purely used mechanical signals for human locomotion mode recognition. For instance, the study [14] combined the IMU sensors on an active pelvic orthosis and the foot pressure sensors for gait mode recognition. The designed algorithm was an event-based fuzzy-logic structure triggered by the foot pressure sensors. The study [15] identified different gait modes with the hip joint angles measured from the encoder of the hip joint exoskeleton. The designed algorithm was a multilayer perceptron neural network. The study [14] conducted a real-time locomotion mode recognition with IMU signals when wearing the active pelvic orthosis (APO [8]). The machine learning-based algorithms were trained and tested onboard. The studies mentioned above produced accurate recognition results on various locomotion mode tasks. However, for hip joint exoskeleton control, burdensome calibration for different individuals and additional sensor nodes on the human body still limited practical applications. For instance, the study overcame the subject-dependent problems with sEMG signals, but the system still required pressure insoles on feet to provide gait event information. The study of our group validated the recognition method with the APO. However, subject-dependent training and calibration were needed before testing procedures.

In this study, we proposed a locomotion mode recognition method based on inertial measurement unit sensors on the hip joint exoskeleton. The designed fuzzy-logic-based algorithm can overcome the subject-dependent parameters in data training, which does not require training for each subject before uses. Besides, no additional sensors are required on the human body, increasing the convenience in practical applications. We preliminarily evaluated the proposed method with an APO on the locomotion mode and locomotion transition recognition on multiple subjects.

\section{Experimental Setups}

2.1. Hip Joint Exoskeleton. In this study, we used an active pelvic orthosis (APO) developed by the research group of Scuola Superiore Sant'Anna (SSSA) [8]. The lightweight exoskeleton can provide assistive torque in the sagittal plane to the hip joints (see Figure 1). The APO was designed with a serial elastic structure based on torsional springs, and the torque was transmitted to the joints with two lightweight carbon fiber-made links (driving part in Figure 1). A C-shaped (in the coronal plane) structure combined with the bandages fixed the exoskeleton to the waist and pelvis of the user, keeping it stable on the human body. Two orthotic shells were connected to the carbon fiber-made links and fixed on the thighs with bandages. The torque was applied to the human body through the shells. There were 3 degree-of-freedoms (DoFs) for each leg, two passive (hip adduction/abduction and pelvic tilting), and one active (flexion/extension) [8]. The passive DoFs ensured the stability of the whole system during ambulation. The core of the actuation system of APO was the DC motors with gearboxes (80:1 reduction ratio). The torsional spring was placed on the axis of the flexion/extension of the exoskeleton, between the DC motor (gearbox) and the carbon fiber-made link. The basic calculation of the interaction torque was achieved by the torsional spring constant and the relative position of the encoders [8]. The interaction torques between the human body and the exoskeleton lead to the deformation of the spring. With the integrated encoders and the stiffness of the torsional spring, the control system of the APO can calculate the interaction torques between the human body and the DC motor.

The control strategy of APO was hierarchical control. The low-level controller was the torque control. The interaction torques calculated from the encoders served as the feedback of the control loop. The control output determined the applied torque on the human body. There were zero-torque mode and assistive-torque mode. For the zero-torque mode, the desired interaction torques between the legs and the exoskeleton were zero. In the assistive-torque mode, the controller's commanded torque was a predefined curve (in one stride). The high-level controller was an adaptive oscillator(AOs-) based controller, which used a set of adaptive oscillators to track the phase of one gait cycle continuously. The input of the AO-based controller was the encoder signals representing the hip joint angle information, and the output of the controller was the gait phase at time $t$ and the corresponding anticipated torque. One merit of the AO-based controller was the continuous estimation of gait phases with robustness to different walking speeds [16].

2.2. Sensing System. We implemented an IMU board on each leg (see Figure 1). The raw signals of the IMU board included 3 -axis accelerations and 3-axis gyroscopes. There was a microcontrol unit (MCU) on the board, i.e., ATMEGA328. The MCU calculated the pitch angle and the roll angle (global frame of the Cartesian system) with the acceleration and gyroscope signals. The board was fixed on the cuff of the exoskeleton through a connector (3D printed). The pitch angle 


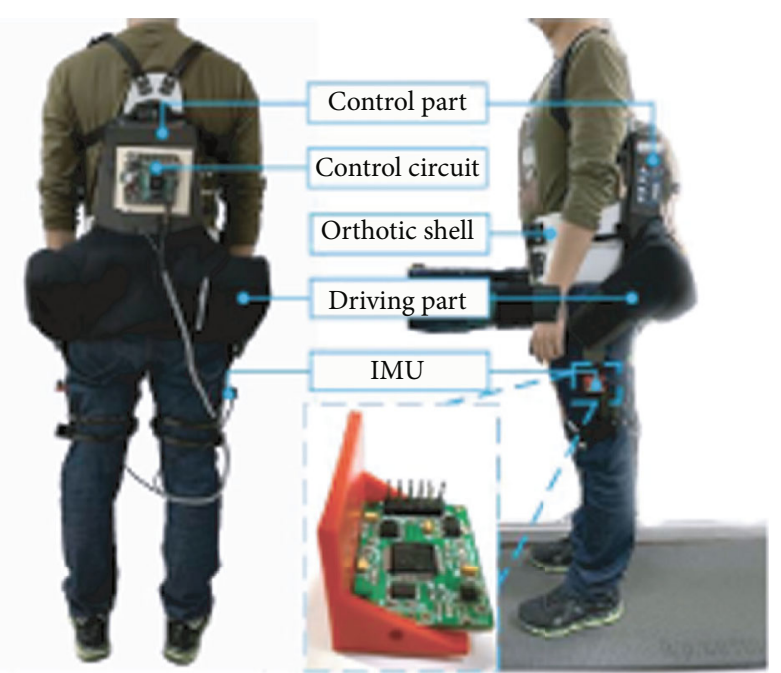

FIgURE 1: The hardware of the system, including the active pelvic orthosis (APO) and the IMU for measurement.

of the IMU corresponded to the flexion/extension of the hip joint. The update rate of the tilt angles was $100 \mathrm{~Hz}$.

The data of the IMU boards were transmitted to a control circuit on the back of APO. The control circuit synchronized the data of APO and the IMU boards via Universal Synchronous Asynchronous Receiver Transmitter (USART). The control circuit integrated a WIFI module. The data of the IMU sensors and the states of APO were transmitted to a host computer wirelessly in each $10 \mathrm{~ms}$. A graphic user interface on the computer was designed with MATLAB R2016b to control the data sequence and store the data.

2.3. Experimental Protocol. In this study, we recruited three healthy subjects. They had an average age of 27.3 years, an average height of $173.7 \mathrm{~cm}$, and an average weight of $67.3 \mathrm{~kg}$. Each subject wore the APO, as shown in Figure 1 in the experiment. In this experiment, we recorded 5 locomotion modes and 8 locomotion transitions. The locomotion modes included standing (St), level walking (LW), stairs ascending (SA), and stairs descending (SD). The locomotion transitions included $\mathrm{St} \longleftarrow \longrightarrow \mathrm{LW}, \mathrm{LW} \longleftarrow \longrightarrow \mathrm{SA}$, and $\mathrm{LW} \longleftarrow \mathrm{SD}$. Each subject performed 4 tasks of ambulation to cover all the locomotion modes and transitions (see Figure 2). For task 1, there were 2 stride cycles of LW and 7 stride cycles of $\mathrm{SA}(\mathrm{St} \longrightarrow \mathrm{LW} \longrightarrow \mathrm{SA} \longrightarrow \mathrm{St}$ ). For task 2 , there were 7 stride cycles of SD and 2 stride cycles of LW $(\mathrm{St} \longrightarrow \mathrm{SD} \longrightarrow \mathrm{LW} \longrightarrow \mathrm{St}$ ). For task 3 , there were 3 stride cycles of LW and 5 stride cycles of SD $(\mathrm{St} \longrightarrow \mathrm{LW} \longrightarrow \mathrm{SD}$ $\longrightarrow \mathrm{St}$ ). For task 4 , there were 5 stride cycles of SA and 3 stride cycles of $\mathrm{LW}(\mathrm{St} \longrightarrow \mathrm{SA} \longrightarrow \mathrm{LW} \longrightarrow \mathrm{St}$ ). The tasks in the experiments were shown in Figure 2. The number of stride cycles was shown in Table 1. In our study, to mimic the locomotion in daily activities, we allowed the subjects to perform the locomotion modes at their favorite paces. Therefore, the number of gait cycles of the subjects was different. Three subjects performed different task repetitions. Subject 1 performed 9 repetitions for each task, including LW with

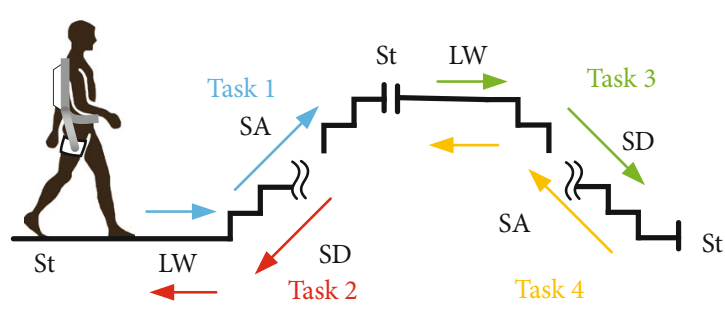

FIgURe 2: The tasks in the experiments. St denotes standing, LW is short for level walking, SA is short for stair ascending, and SD is short for stair descending. The arrows indicate ambulation direction. The tasks are denoted with different colors.

90 gait cycles, SA with 108 gait cycles, SD with 108 gait cycles, and 9 repetitions for each locomotion transition $(\mathrm{LW} \longrightarrow \mathrm{SA}$, $\mathrm{SA} \longrightarrow \mathrm{LW}$, gait initiation/termination). Subject 2 performed 5 repetitions for each task, including LW with 50 gait cycles, SA with 60 gait cycles, SD with 60 gait cycles, and 5 repetitions for each locomotion transition. Subject 3 performed 6 repetitions for each task, including LW with 60 gait cycles, SA with 72 gait cycles, SD with 72 gait cycles, and 6 repetitions for each locomotion transition.

\section{Recognition Method}

3.1. Cascaded Recognition Method. The locomotion recognition method was designed based on the signal features acquired in the IMUs of both legs. The recognition method is cascaded, which firstly (first layer) classifies the static mode (St) and dynamic modes (LW, SA, SD) and secondly (second layer) identifies the corresponding dynamic locomotion modes (see Figure 3). In the second layer, we designed a fuzzy-logic-based algorithm. There were two membership function sets in the fuzzy-logic-based algorithms, one for each leg. The input of the membership functions was the data pair of peak-valley values detected from the thigh angles. Therefore, in the second layer, we firstly identified the peaks and valleys and secondly calculated the fuzzy-logic membership functions. During the locomotion transitions, there are different leading legs. The procedures of two legs worked independently in the recognition process.

In the cascaded recognition method, the first step is to distinguish between the static locomotion mode and the dynamic modes. As there were no gait patterns during standing, the signal profiles were much different from that of ambulation modes. We extracted time-domain features to represent the signal profiles of standing and other locomotion modes. We firstly segmented the data (pitch angles and accelerations) with a $100 \mathrm{~ms}$ (10 samplings) sliding window. We calculated the standard deviation on the windows of the left leg's pitch angles $\operatorname{std}\left(\theta_{L}\right)$ and the sum of absolute values of 3-axis accelerations sum(acc). Additionally, we compared the angular difference of the two legs in the sagittal plane, expressed as $\theta_{\text {relative }}=\left|\theta_{L}-\theta_{R}\right| . \theta_{R}$ is the right thigh's pitch angle, and $\theta_{L}$ is the left thigh's pitch angle. $\theta_{\text {relative }}$ is the relative pitch angle between the left thigh and right thigh. 
TABLE 1: The stride cycle number in each task.

\begin{tabular}{lcccc}
\hline & Task 1 & Task 2 & Task 3 & Task 4 \\
\hline LW (stride) & 2 & 2 & 3 & 3 \\
SA (stride) & 7 & 0 & 0 & 5 \\
SD (stride) & 0 & 7 & 5 & 0 \\
\hline
\end{tabular}

The first layer recognition was achieved by comparing the threshold-based conditions. The logic was expressed as

$$
\begin{aligned}
& \operatorname{std}\left(\theta_{L}\right)>T h_{\text {std }} \text { or }\left|\theta_{L}-\theta_{\text {init }}\right|>T h_{\theta} \text { or }\left|\theta_{R}-\theta_{\text {init }}\right| \\
& \quad>T h_{\theta} \text { or } \operatorname{sum}(a c c)>T h_{\text {Acc }} \text { and } \theta_{\text {relative }}>\theta_{\text {static. }}
\end{aligned}
$$

If the logic condition was satisfied, the mode would be recognized as dynamic modes. Otherwise, it was classified as the static mode (St). $\theta_{\text {init }}$ is the initial pitch angle for both thighs which is close to 0 .

In the above logic condition, $\mathrm{Th}_{\text {std }}$ was the standard deviation threshold for the sliding window which was selected as $0.5^{\circ} . \mathrm{Th}_{\theta}$ was the pitch angle threshold for both legs which was selected as $8^{\circ} . \theta_{\text {static }}$ was the threshold for the static mode which was selected as $10^{\circ}$. Th $\mathrm{Acc}_{\text {cc }}$ was the threshold for acceleration which was chosen as 500 .

3.2. Detecting the Extreme Values. The second layer was to further separate the data into corresponding dynamic locomotion modes (i.e., LW, SA, and SD). We designed a fuzzylogic-based algorithm to classify the locomotion modes. Each input was a $2 \times 1$ vector including the peak and valley of the pitch angles. We designed an algorithm find_peak() to detect the peaks and valleys of the IMU signals. The most recent true peak and valley values found by find_peak() would be put in a $2 \times 1$ buffer as the input of the subsequent fuzzylogic-based algorithms. Taking finding the left leg's peak values for example (diagram see Figure 4), we firstly predefined thresholds for peak value $\mathrm{Th}_{- \text {peak }}$ and time interval Th-interval. $\theta_{L}(t)$ was the pitch angle of the left leg at time $t$. We used $P(i)$ to represent the $i_{\text {th }}$ peak value found in (pseudo) real-time. Secondly, the past 21 samples before time $t$ were compared (i.e., $\left.\theta_{L}(t), \theta_{L}(t-1), \cdots, \theta_{L}(t-20)\right)$. If $\theta_{L}(t$ $-10)$ is larger than all the 10 numbers backward $\left(\theta_{L}(t-20), \theta_{L}(t-19), \cdots \theta_{L}(t-11)\right.$ and all the 10 numbers forward $\left(\theta_{L}(t), \theta_{L}(t-1) \cdots \theta_{L}(t-9)\right)$, we set $P(i)=\theta_{L}(t-$ 10). If the absolute value of peak value $P(i)$ minus the initial value of pitch angle $\theta_{L}(0)$ was larger than $\mathrm{Th}_{- \text {peak }}$, we would consider the peak value $P(i)$ as an outlier which would be discarded. Otherwise, the $P(i)$ would be treated as a peak candidate. Because of the existence of false peak values created by the noise, we compared peak candidate's location location $P(i)$ with that of the latest candidate location_P(i-1). We set the time interval threshold $\mathrm{Th}_{- \text {interval }}$ for two adjacent peak values. If the time interval between these two peak values was smaller than $T h_{\text {-interval }}$, we would assume that one of the two candidate peak values was false. The candidate with the smaller value was aborted. We set a decision flag for the algorithm. The decision flag would be activated if a true peak and a true valley were detected. The peak-valley pair was then inputted to the subsequent fuzzy-logic-based algorithm, and then the flag would be deactivated. The procedure of detecting valleys and the values of the other leg was the same.

\subsection{Fuzzy-Logic-Based Recognition Method. We designed a} fuzzy-logic-based method to separate between the locomotion modes of LW, SA, and SD. As mentioned above, the input of the fuzzy-logic algorithm was a 2-dimensional (2D) vector containing the latest detected peak and valley (one leg), represented as $\theta p$ and $\theta v$, respectively. The maximum/minimum values revealed the characteristics of different locomotion modes. For instance, the $\theta p$ values of SA were larger than that of LW and SD, as the hip joint angles were larger in flexion when ambulating upward. The valleys of the thigh pitch angles also demonstrated similar features. During the LW and SD mode, $\theta v$ values were at the same level, while they would decrease during the SA mode because the stair-ascending locomotion contains a kicking-back movement in which the hanging leg could reach the lowest pitch angle without the constraint of the stairs. During LW and $\mathrm{SD}, \theta v$ would be limited by the ground and the stairs. We visualized the characteristics in Figure 5. The distribution of maximum-minimum of different locomotion modes could be separated apart.

We designed multivariate membership functions to classify the three locomotion modes. The membership function calculates the membership value of the event-based feature belonging to the target mode. The output range of membership is $(0,1]$, where 1 is the maximum membership of the model. The membership functions were calculated in parallel with the signals of two legs. For the signals of each leg, we defined three membership functions, one for each locomotion mode. The function was expressed as

$$
f_{i}=\frac{k_{i}}{2 \pi\left|\sum_{i}\right|^{1 / 2}} e^{-\left[\frac{1}{2}\left(C_{i}-\bar{X}_{i}\right)^{T} \Sigma_{i}^{-1}\left(C_{i}-\bar{X}_{i}\right)\right]}, i=1,2,3,
$$

where $i$ denoted the mode's number, $k_{i}$ was the scale factor, $C_{i}=\left(\theta_{p i}, \theta_{v i}\right)^{T}$ was the input vector including the detected peak and valley, and $\bar{X}_{i}=\left(\mu_{p i}, \mu_{v i}\right)^{T}$ was the central point of the membership function. In our study, the point was represented as the mean value of the training data sets. $\Sigma_{i}$ was the covariance matrix representing the data distribution. After calculating three membership functions of LW, SA, and SD, respectively, the algorithm proceeded to calculate the maximal membership of the target mode:

$$
\text { Target mode }=\arg \max _{i}\left(f_{i}\right) .
$$

Figure 5 shows that 2-dimensional space three membership functions created three oval shape regions, whose center coordinates were the mean value of three membership functions $\left(\bar{X}_{i}\right)$. In our study, the parameters $\bar{X}_{i}$ and $\Sigma_{i}$ were fitted with the training data set (described in detail below).

3.4. Synchronization of the Recognition Decisions. There were inertial sensors on both thighs. The fuzzy-logic-based recognition method worked in parallel for the left leg and the right 


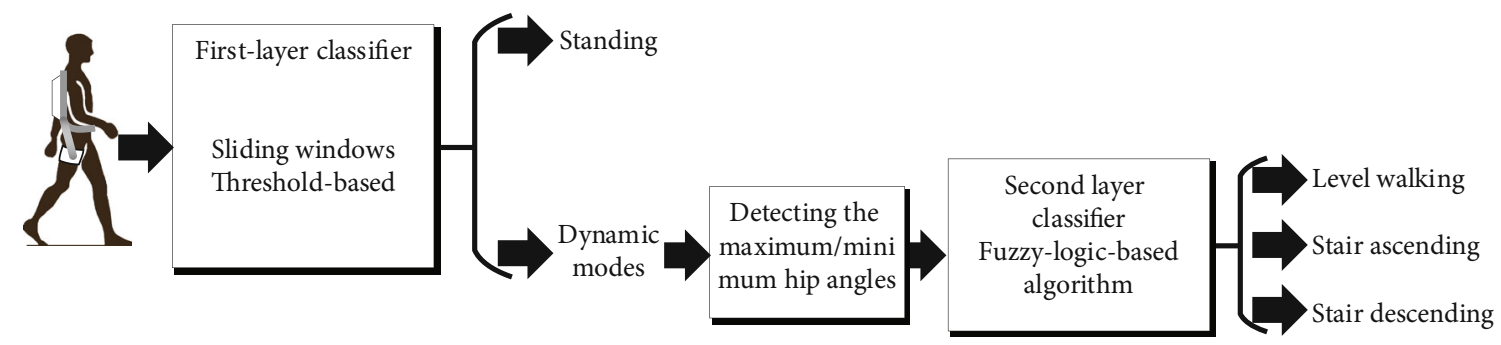

FIgure 3: The diagram of the recognition method. The first layer was to distinguish dynamic modes from standing (St). The second layer was designed to classify the dynamic modes, and there were three dynamic modes (LW, SA, and SD).

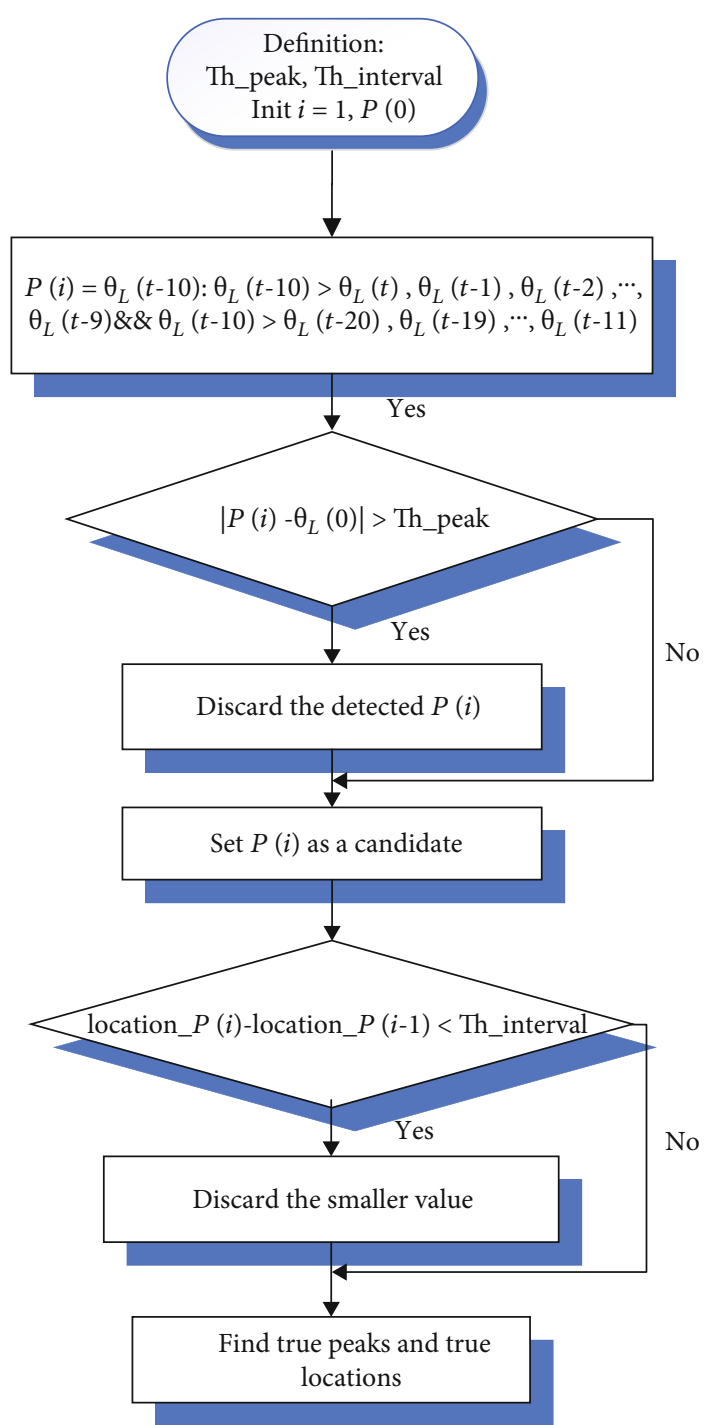

FIGURE 4: The flow chart of finding a peak value from the thigh pitch angles.

leg. The recognition decisions were then synchronized to minimize the errors in locomotion transitions. In our cascaded recognition method, the first layer was to distinguish between St and dynamic modes. The transitions were gait initiation and gait termination. For gait initiation recognition
(St $\longrightarrow$ othermodes), the first recognized transition was deemed to be the results, which were expressed as

$$
\begin{aligned}
t_{i}= & \min \left[\left(\text { right legs's transition time point } t_{R}\right),\right. \\
& \left.\cdot\left(\text { left leg's transition time } t_{L}\right)\right],
\end{aligned}
$$

where $t_{i}$ was the timing point of the detected transition. For gait termination recognition, the last recognized transition was deemed to the recognition results. The timing point $t_{t}$ was expressed as

$$
\begin{aligned}
& t_{t}=\max \left[\left(\text { right leg }^{\prime} \text { s transition time point } t_{R}\right),\right. \\
& \text { - (left leg's transition time } \left.\left.t_{L}\right)\right] \text {. }
\end{aligned}
$$

For the second layer recognition, the transition timing points $\left(t_{0 d}\right)$ were the first recognized timing points between the left leg's and right leg's results.

\section{Evaluation Method}

4.1. Crossvalidation. We used the crossvalidation method to evaluate the performances. We evaluated the performance with $1: 2$ intersubject crossvalidation and $1: 1$ intrasubject crossvalidation. In the 1:1 intrasubject crossvalidation, each subject's data were divided into two sets with the same sizes. The first data were used for training, and the second set for testing. The procedure was repeated with the second data set for training and the first set for testing. The results of the two tests were averaged as the result of the subject. In the 1:2 intersubject crossvalidation, we used the data of one subject for training and the data of the other subjects for testing. In the training procedure, the parameters of the fuzzy-logic-based algorithms were fitted.

4.2. Recognition Accuracy. The first metric for evaluating the performance was the recognition accuracy.

In the first layer, the recognition decisions (St and other dynamic modes) were continuously calculated in each sample. The recognition accuracy (recognition accuracy 1) was defined as

$$
\operatorname{recognition} \operatorname{accuracy} 1(i)=\frac{N \operatorname{correct} 1(i)}{N \operatorname{total} 1(i)}
$$




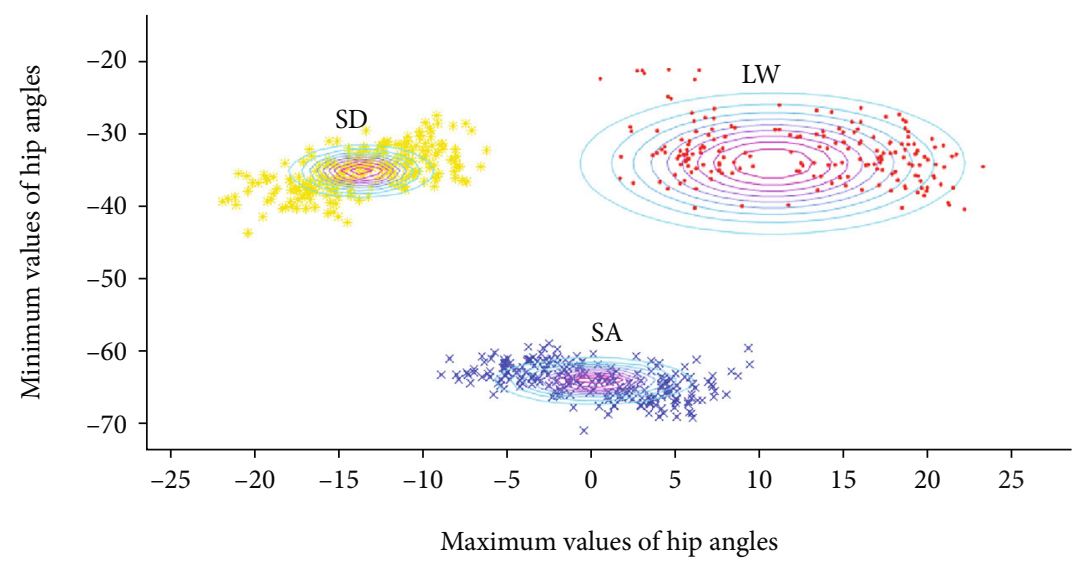

FIGURE 5: The distribution of the data of LW, SA, and SD (denoted by the dots) and the calculated membership function (represented by the ellipses). The data of LW, SA, and SD were represented as the yellow dots, the red dots, and the blue dots, respectively. The data were collected from subject 1 .

where $i$ was the subject's number, $N$ correctl was the number of correctly recognized decisions, and Ntotall was the total number of decisions.

In the second layer, the recognition decisions were calculated in each extreme point being detected (peak and valley). The recognition accuracy of the second layer was defined as

$$
\operatorname{recognition} \operatorname{accuracy}(i)=\frac{N \operatorname{correct}(i)}{N \operatorname{total}(i)},
$$

where $i$ was the number of subjects, $N$ correct was the number of correctly recognized gait cycles, and Ntotal was the total number of gait cycles.

4.3. Confusion Matrix. We used the confusion matrix to illustrate the recognition performance of each locomotion mode. The details of the definition can be found in [17].

4.4. Time Delay of the Locomotion Transitions. Another metric for evaluating the performance was the time delay. There were three critical timing points in each transition period, i.e., the timing point when the data changed from St to dynamic modes (gait initiation, $t_{0 i}$ ), the timing point when the data changed from dynamic modes to St (gait termination, $t_{0 t}$ ), and the timing point when the data changed from one dynamic mode to another $\left(t_{0 d}\right)$.

The reference transition time was determined by labels. In our cascaded recognition method, the first layer was to separate between standing and dynamic modes. We manually labeled the data as standing and dynamic modes by IMU signals. If the pitch angles exceeded a threshold compared with that of standing, the data would be labeled as dynamic modes, and $t_{0 i}$ was defined as the reference of the gait initiation transition time between standing and dynamic modes, while $t_{0 t}$ was defined as the reference of the gait termination transition time between dynamic and standing modes. For the second layer recognition, the reference transition time was labeled based on the gait events detected by the foot pressure insoles. $t_{0 d}$ was defined as the reference of the transition time between two dynamic modes. $t_{0 d}$ would be labeled as the middle point of the swing phase. The labeling method motion transitions were the same as that of existing-related studies $[18,19]$.

The time delay (Td_init) of gait initiations was defined as the difference between the recognized timing point $t_{i}$ and the reference transition time of gait initiation $t_{0 i}$, expressing as

$$
\text { Td_init }=t_{i}-\mathrm{t}_{0 i} \text {. }
$$

Similarly, the timed delay (Td_terminal) of gait terminations was expressed as

$$
\text { Td_terminal }=t_{t}-t_{0 t} \text {, }
$$

where $t_{t}$ was the recognition transition time of gait termination, and $t_{0 t}$ was the reference transition time of gait termination.

The time delay (Td_dynamic) of the dynamic modes was expressed as

$$
\text { Td_dynamic }=t_{d}-t_{0 d} \text {, }
$$

where $t_{d}$ was the recognition transition time of dynamic modes, and $t_{0 d}$ was the reference transition time of dynamic modes. The positive value presented the delay of recognition, and the negative value represented the advance of recognition, shown in Figure 6.

\section{Results}

5.1. Recognition Accuracy. In this section, we showed the recognition accuracy for both the first and second layer classifiers. The first layer recognition was designed to distinguish dynamic modes from standing (St). The second layer recognition was designed to classify three dynamic modes (LW, $\mathrm{SA}$, and SD).

As for the first layer (classification between dynamic modes and St), the recognition accuracy for each subject was $92.18 \%, 93.00 \%$, and $90.45 \%$, respectively. The average recognition accuracy was $91.88 \%$. As for the second layer 

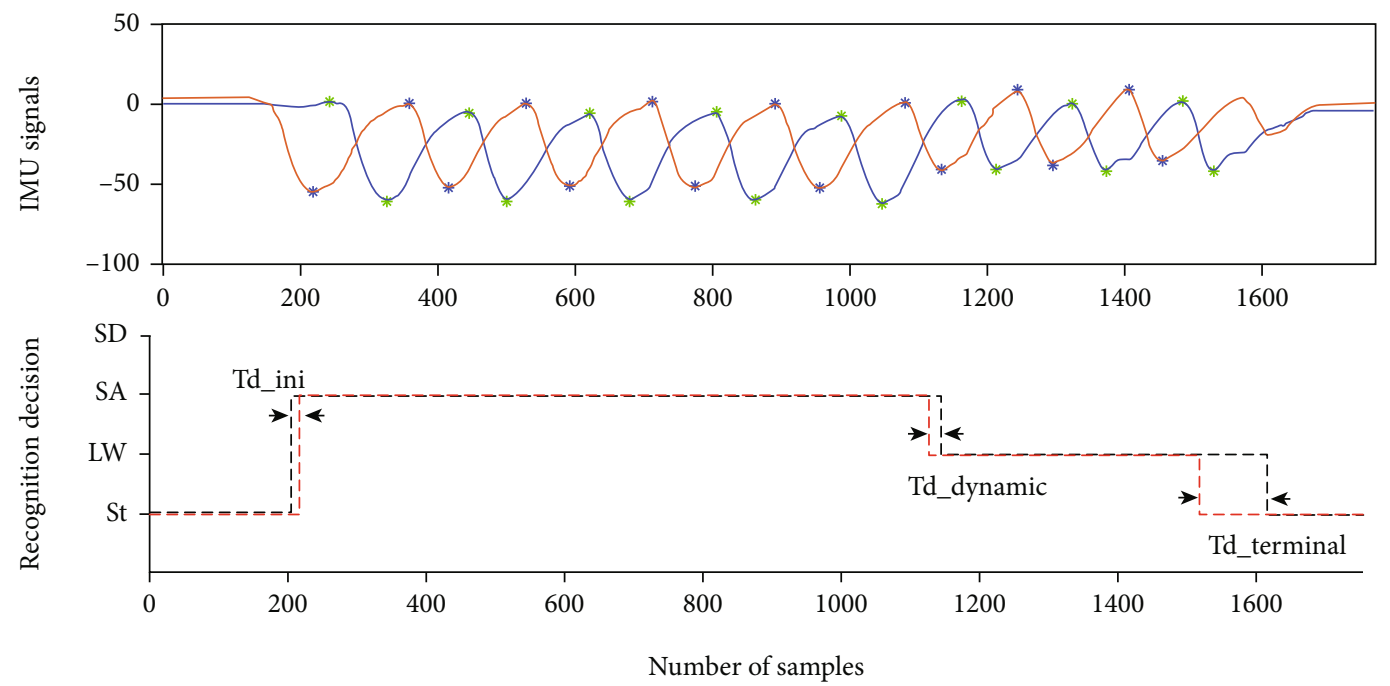

-- - Right leg
-- - Reference

Figure 6: The pseudo real-time recognition decisions. The upper subplot is the raw pitch angles and the detected extreme points. The bottom subplot shows the recognition decisions and the reference labels.

(classification between LW, SA, and SD), the fuzzy-logicbased method produced accurate recognition decisions in locomotion mode tasks (the case result see Figure 6). The recognition accuracy was higher than 0.89 for most of the evaluations (intersubject shown in Table 2 and intrasubject shown in Table 3). In Tables 2 and 3, each row represented the subject number used for training, and each column represented the testing data (the subject used for testing). The offdiagonal results in Table 2 were the intersubject recognition accuracies, while the diagonal results in Table 3 denoted the accuracies of the intrasubject crossvalidation.

In the 1:2 intersubject validation (see in Table 2), the membership functions trained with subject3's dataset had the best performance, with the highest accuracy, 95.51\%. The average recognition accuracy for each subject trained with different datasets was $91.54 \%, 92.80 \%$, and $95.14 \%$ for subject 1 , subject 2 , and subject 3 , respectively. The lowest accuracy $(89.84 \%)$ occurred in subject 1 trained with subject 2's dataset. Subject 3's average recognition accuracy showed the best performance, $95.14 \%$.

In Table 3 (intrasubject crossvalidation), recognition accuracies showed a similar pattern but slightly decreased compared with intersubject validation. The lowest accuracy, $86.80 \%$, occurred in subject 1 trained with subject3's dataset. The average testing set recognition accuracy of three subjects trained with the same training dataset was $92.98 \%, 93.26 \%$, and $91.16 \%$, respectively, with subject 1 , subject 2 , and subject3's training dataset. The average testing set recognition accuracy for each subject trained with different training datasets was $87.62 \%, 94.27 \%$, and $95.51 \%$, respectively.

In Tables 4 and 5, we presented the recognition accuracy for each task. From the experiment results, the lowest recognition accuracy for each subject's results usually occurred in task $2(\mathrm{St} \longrightarrow \mathrm{SD} \longrightarrow \mathrm{LW} \longrightarrow \mathrm{St}$ ), while the recognition algorithm usually performed better in task 1 and task 4 .
TABLE 2: Training/testing with whole data of a subject $(1: 2$ intersubject validation).

\begin{tabular}{lccc}
\hline & Subject 1 & Subject 2 & Subject 3 \\
\hline Subject 1 & - & $90.39 \%$ & $94.76 \%$ \\
Subject 2 & $89.84 \%$ & - & $95.51 \%$ \\
Subject 3 & $93.23 \%$ & $95.20 \%$ & - \\
\hline
\end{tabular}

TABle 3: Training/testing with half data of a subject $(1: 1$ intrasubject validation).

\begin{tabular}{lccc}
\hline & Subject 1 & Subject 2 & Subject 3 \\
\hline Subject 1 & $88.29 \%$ & $95.42 \%$ & $95.22 \%$ \\
Subject 2 & $87.76 \%$ & $95.31 \%$ & $96.70 \%$ \\
Subject 3 & $86.80 \%$ & $92.07 \%$ & $94.60 \%$ \\
\hline
\end{tabular}

5.2. Time Delay of Locomotion Transitions. We investigated the time delay during locomotion transitions trained/tested with half the data of a subject. We calculated the time difference between the recognized locomotion transitions and the referenced ones. We defined the time latency of gait initiations as Td_init, gait terminations as Td_terminal, and the dynamic transition as Td_dynamic. The unit of the results was ms.

From Table 6, we can see that the average time delay of gait initiations for each subject was $1077.3 \mathrm{~ms}, 812.8 \mathrm{~ms}$, and $268.2 \mathrm{~ms}$ and the average time delay of gait terminations was $787.5 \mathrm{~ms}, 315.5 \mathrm{~ms}$, and $29.2 \mathrm{~ms}$. The average time delay for all the subjects was $554.4 \mathrm{~ms}$. Subject 3 has the lowest time delay for both gait initiations and gait terminations $(268.2 \mathrm{~ms}$ and $29.2 \mathrm{~ms}$ ). Also, we can find that large time delays occurred in task 2 and task 3 frequently. 
TABLE 4: Recognition accuracy of $1: 2$ intersubject validation.

\begin{tabular}{|c|c|c|c|c|c|c|c|c|c|c|}
\hline & \multicolumn{3}{|c|}{ Subject 1} & \multicolumn{3}{|c|}{ Subject 2} & \multicolumn{3}{|c|}{ Subject 3} \\
\hline & & $\begin{array}{l}\text { Number of } \\
\text { error steps }\end{array}$ & $\begin{array}{l}\text { Number of } \\
\text { total steps }\end{array}$ & $\begin{array}{c}\text { Accuracy } \\
\text { rate }\end{array}$ & $\begin{array}{l}\text { Number of } \\
\text { error steps }\end{array}$ & $\begin{array}{l}\text { Number of } \\
\text { total steps }\end{array}$ & $\begin{array}{c}\text { Accuracy } \\
\text { rate }\end{array}$ & $\begin{array}{l}\text { Number of } \\
\text { error steps }\end{array}$ & $\begin{array}{c}\text { Number of } \\
\text { total steps }\end{array}$ & $\begin{array}{c}\text { Accuracy } \\
\text { rate }\end{array}$ \\
\hline \multirow{4}{*}{ Subject 1} & Task 1 & - & - & - & 2 & 95 & $98.48 \%$ & 1 & 109 & $99.08 \%$ \\
\hline & Task 2 & - & - & - & 20 & 85 & $76.47 \%$ & 12 & 102 & $88.24 \%$ \\
\hline & Task 3 & - & - & - & 10 & 74 & $86.49 \%$ & 7 & 93 & $92.47 \%$ \\
\hline & Task 4 & - & - & - & 0 & 82 & $100.00 \%$ & 1 & 97 & $98.97 \%$ \\
\hline \multirow{4}{*}{ Subject 2} & Task 1 & 15 & 174 & $91.38 \%$ & - & - & - & 1 & 109 & $99.08 \%$ \\
\hline & Task 2 & 21 & 176 & $88.07 \%$ & - & - & - & 10 & 102 & $90.20 \%$ \\
\hline & Task 3 & 18 & 175 & $89.71 \%$ & - & - & - & 6 & 93 & $93.55 \%$ \\
\hline & Task 4 & 15 & 154 & $90.26 \%$ & - & - & - & 1 & 97 & $98.97 \%$ \\
\hline \multirow{4}{*}{ Subject 3} & Task 1 & 18 & 174 & $89.66 \%$ & 3 & 132 & $97.73 \%$ & - & - & - \\
\hline & Task 2 & 19 & 176 & $89.20 \%$ & 8 & 85 & $90.59 \%$ & - & - & - \\
\hline & Task 3 & 12 & 175 & $93.14 \%$ & 3 & 74 & $95.95 \%$ & - & - & - \\
\hline & Task 4 & 18 & 154 & $88.31 \%$ & 2 & 82 & $97.56 \%$ & - & - & - \\
\hline
\end{tabular}

TABle 5: Recognition accuracy of $1: 1$ intrasubject crossvalidation.

\begin{tabular}{|c|c|c|c|c|c|c|c|c|c|c|}
\hline & \multicolumn{3}{|c|}{ Subject 1} & \multicolumn{3}{|c|}{ Subject 2} & \multicolumn{3}{|c|}{ Subject 3} \\
\hline & & $\begin{array}{l}\text { Number of } \\
\text { error steps }\end{array}$ & $\begin{array}{l}\text { Number of } \\
\text { total steps }\end{array}$ & $\begin{array}{c}\text { Accuracy } \\
\text { rate }\end{array}$ & $\begin{array}{l}\text { Number of } \\
\text { error steps }\end{array}$ & $\begin{array}{l}\text { Number of } \\
\text { total steps }\end{array}$ & $\begin{array}{l}\text { Accuracy } \\
\text { rate }\end{array}$ & $\begin{array}{l}\text { Number of } \\
\text { error steps }\end{array}$ & $\begin{array}{l}\text { Number of } \\
\text { total steps }\end{array}$ & $\begin{array}{l}\text { Accuracy } \\
\text { rate }\end{array}$ \\
\hline \multirow{4}{*}{ Subject 1} & Task 1 & 1 & 79 & $98.73 \%$ & 1 & 37 & $97.30 \%$ & 0 & 54 & $100.00 \%$ \\
\hline & Task 2 & 15 & 83 & $81.93 \%$ & 5 & 32 & $84.38 \%$ & 6 & 56 & $89.29 \%$ \\
\hline & Task 3 & 18 & 78 & $76.92 \%$ & 0 & 28 & $100.00 \%$ & 3 & 47 & $93.62 \%$ \\
\hline & Task 4 & 3 & 68 & $95.59 \%$ & 0 & 33 & $100.00 \%$ & 1 & 49 & $97.96 \%$ \\
\hline \multirow{4}{*}{ Subject 2} & Task 1 & 9 & 79 & $88.61 \%$ & 1 & 37 & $97.30 \%$ & 0 & 54 & $100.00 \%$ \\
\hline & Task 2 & 12 & 83 & $85.54 \%$ & 4 & 32 & $87.50 \%$ & 5 & 56 & $91.07 \%$ \\
\hline & Task 3 & 10 & 78 & $87.18 \%$ & 1 & 28 & $96.43 \%$ & 2 & 47 & $95.74 \%$ \\
\hline & Task 4 & 7 & 68 & $89.71 \%$ & 0 & 33 & $100.00 \%$ & 0 & 49 & $100.00 \%$ \\
\hline \multirow{4}{*}{ Subject 3} & Task 1 & 10 & 79 & $87.34 \%$ & 1 & 37 & $97.30 \%$ & 0 & 54 & $100.00 \%$ \\
\hline & Task 2 & 10 & 83 & $87.95 \%$ & 7 & 32 & $78.13 \%$ & 4 & 56 & $92.86 \%$ \\
\hline & Task 3 & 7 & 78 & $91.03 \%$ & 2 & 28 & $92.86 \%$ & 2 & 47 & $95.74 \%$ \\
\hline & Task 4 & 13 & 68 & $80.88 \%$ & 0 & 33 & $100.00 \%$ & 5 & 49 & $89.80 \%$ \\
\hline
\end{tabular}

For Table 7, we can see that the average Td_dynamic for each subject was $685.1 \mathrm{~ms}, 541.8 \mathrm{~ms}$, and $394.0 \mathrm{~ms}$. The lowest average Td_dynamic also occurred in subject 3 . Also, we can see that the large Td_dynamic usually occurred in task 3 .

The average gait cycle was $1897.9 \mathrm{~ms}$, for which the average time delay of average Td_init, average Td_terminal, and average Td_dynamic was $549.5 \mathrm{~ms}$ accounted for $28.95 \%$ of a gait cycle.

\section{Discussion}

6.1. Recognition Performances. In this study, we designed and evaluated the fuzzy-logic-based method for locomotion mode/transition recognition with a hip joint exoskeleton. The method only relied on the inertial sensors integrated into the exoskeleton, and no additional sensors were required on the human body. Compared with the previous works using the same exoskeleton $[14,20]$, one improvement of our cur- rent study was that we simplified the setups of the sensing approaches in both training and testing procedures. The simplification in sensors can reduce the time needed to calibrate the recognition procedure in practical applications.

There are many studies on IMU-based locomotion mode recognition. The performances are determined by the factors, including the sensor setups (sensors' number, sensing positions), the robotic devices (exoskeletons, prostheses), and the processing algorithms. The evaluation method also influenced the numeric recognition results. For instance, the recent studies on IMU-based locomotion mode recognition achieved $>95 \%$ average recognition accuracies with intrasubject crossvalidation $[21,22]$. The sensor setups are quite different from ours. The study mounted an IMU board on the amputated foot for terrain identification [21], while the study fixed the IMU boards on the shanks, the waist, and wrists [22].

In our study, the IMU boards were fixed on the thighs of the subjects. The target robot platform is the hip exoskeleton. 
TABLE 6: The initial and terminal transition time latency.

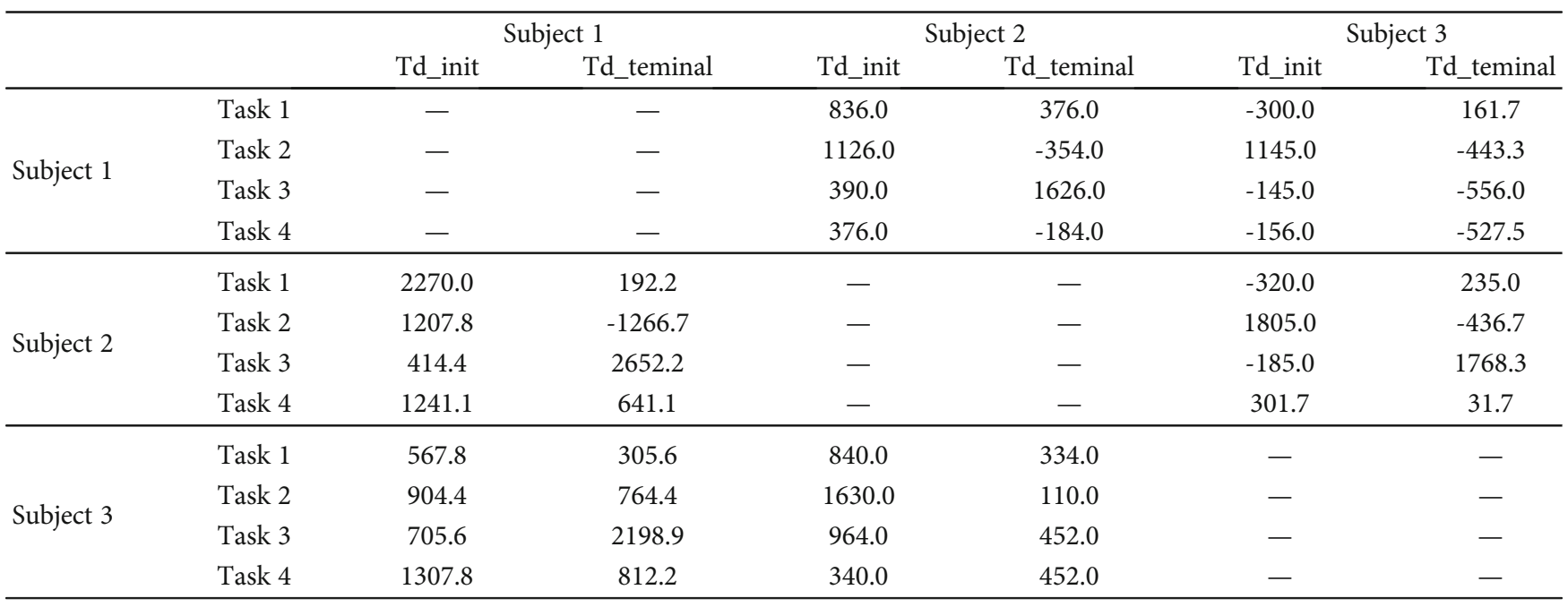

TABle 7: The dynamic transition time latency.

\begin{tabular}{|c|c|c|c|c|}
\hline & \multicolumn{3}{|c|}{ Td_dynamic } \\
\hline & & Subject 1 & Subject 2 & Subject 3 \\
\hline \multirow{4}{*}{ Subject 1} & $\mathrm{LW} \longrightarrow \mathrm{SA}$ & - & 444.0 & 230.0 \\
\hline & $\mathrm{SD} \longrightarrow \mathrm{LW}$ & - & 136.0 & -405.0 \\
\hline & $\mathrm{LW} \longrightarrow \mathrm{SD}$ & - & 1696.0 & 1815.0 \\
\hline & $\mathrm{Sa} \longrightarrow \mathrm{LW}$ & - & -154.0 & -1.7 \\
\hline \multirow{4}{*}{ Subject 2} & $\mathrm{LW} \longrightarrow \mathrm{SA}$ & 235.6 & - & 230.0 \\
\hline & $\mathrm{SD} \longrightarrow \mathrm{LW}$ & 5.6 & - & -405.0 \\
\hline & $\mathrm{LW} \longrightarrow \mathrm{SD}$ & 235.6 & - & 1690.0 \\
\hline & $\mathrm{Sa} \longrightarrow \mathrm{LW}$ & 687.8 & - & -1.7 \\
\hline \multirow{4}{*}{ Subject 3} & $\mathrm{LW} \longrightarrow \mathrm{SA}$ & 365.6 & 444.0 & - \\
\hline & $\mathrm{SD} \longrightarrow \mathrm{LW}$ & 921.1 & 136.0 & - \\
\hline & $\mathrm{LW} \longrightarrow \mathrm{SD}$ & 2198.9 & 1526.0 & - \\
\hline & $\mathrm{Sa} \longrightarrow \mathrm{LW}$ & 814.4 & 106.0 & - \\
\hline
\end{tabular}

The recognition performances of our study were at the same level as that of the previous studies with similar sensor/robot setups $[14,20]$. In the previous work with the active pelvic orthosis [14], the authors used inertial sensors on the thigh and the foot pressure insoles for seven locomotion mode recognitions. The average accuracies on six healthy subjects achieved over $99 \%$ with locomotion transition tasks. The average time delay during the locomotion transitions was fixed to one step. On the other hand, the authors also claimed the limitations of using additional foot insoles which was not integrated on the exoskeleton. The accuracies decreased to $65.7 \%-91.2 \%$ in different testing data sets if the centre-ofpressure (CoP) information was removed [14]. In our previous works [20], the authors designed machinelearning-based algorithms for locomotion mode recognition. In the study of [23], the authors designed an artificial neural network- (ANN-) based recognition algorithm with the inertial signals (thigh) and the foot pressure signals. The average recognition accuracies were over $98 \%$ with subjectdependent training and testing processes. The calculation time of each recognition decision was less than $1 \mathrm{~ms}$, but the time delay of locomotion transitions was not reported. By comparison, in our study, we produced an average recognition accuracy of $93.16 \%$ with 1 vs. 2 intersubject crossvalidations and $92.46 \%$ with 1 vs. 1 intrasubject crossvalidation.

6.2. Confounding Factors. One key factor that influenced the recognition accuracies was the detection of the peaks and valleys from the inertial signals. As shown in Figure 6, the locomotion modes and transitions were successfully recognized; although, there were misdetections in the extreme values. The output value of the membership functions was determined by the detected values of the extreme points. In the calculated recognition results, the fuzzy-logic-based method could successfully tell apart the locomotion modes as long as the distribution of the extreme points was distinguishable (as shown in Figure 5). In our study, although no signals from the feet were measured, the maximum/minimum angles still showed gait information. The maximum value of the thigh tilt angle usually occurred at the swing phase, which was used to distinguish between the LW and SA. While the minimum angle occurred near the foot-off of one gait cycle, the values were informative to distinguish between LW and SD. The maximum hip flexion/extension angles are highly correlated to the locomotion modes. For instance, the maximum flexion angles of SA were significantly larger than that of LW and SD. The subjects can adjust the patterns intuitively to control the exoskeletons in locomotion transition tasks. The physical significance of the features in our fuzzylogic-based algorithm can accelerate the training/calibration procedure for a novice subject. Another point worth being noted is the intersubject variability in signal profiles and recognition performances. In addition to the difference in motion patterns, the difference in relative positions of the IMU boards on the thigh was another important reason. During the experiments, the IMU boards were fixed on the same positions at the exoskeleton. Due to the different 
anthropometries of the subjects, the relative positions on the thigh were different. In practical applications, the sensors usually are fixed on the exoskeleton. Adjusting the sensor position to keep the same signal profiles across subjects is also impractical. In future real-time control, we will improve the recognition algorithms with the ability of fast calibration to make the trained model quickly update with the new user.

6.3. Influence of the Recognition Performances on Robotic Control. In real-time control of the APO, the hierarchical control framework is usually designed. The high-level controller recognizes the locomotion modes and determines the assistive torque curve of the recognized terrain. The middle-level controller uses adaptive oscillators (AOs) to track the desired torque curve. The low-level controller drives the motors to achieve the force feedback loop.

If there are recognition errors, the desired torque curve for the controller will be different from that needed for the current terrain. The user will move with an inappropriate assistive torque curve. The APO applies assistive torque on the hip joint angle in the sagittal plane. Due to the mechanical design of the APO (2 passive DoFs in the coronal plane and passive compliance), it is less likely that the user will fall caused by the wrong recognition of the locomotion modes. However, in the long-time use, the mismatching between the assistive torque curve and the terrains can increase the metabolic cost (decrease the efficacy of the exoskeleton) and the risks of the fall. If the transition time delay exceeds the starting timing point of the applied assistive torque of one stride, there will be a mismatch between the assistance and the actual locomotion mode. Otherwise, the time delay is acceptable. In our study, the average time delay during the transitions ranges from $300 \mathrm{~ms}$ to $1000 \mathrm{~ms}$, which can cause a mismatch between the assistive torques and current locomotion modes. The impacts on the user are the same as that of the recognition errors.

To quantitatively evaluate the recognition errors, further extensive experiments combing the real-time recognition and exoskeleton controller are needed. In future studies, we will investigate the effects of the errors and time delay with real-time recognition and control.

6.4. Limitations and Future Works. Our current study has some limitations, and the following issues will be addressed in future works. Firstly, the sample size of the subjects was small $(N=3)$. The results were calculated with an offline evaluation. The generalization ability of the fuzzy-logicbased algorithm cannot be extensively evaluated with the small sample size. Due to the individual difference in locomotion patterns and sensor placements, the signal profiles can vary across the subjects. The onboard training and realtime exoskeleton control have yet to be studied. In future works, we will carry out an extensive study on real-time control with onboard training. We will investigate the effects of false detections on control performances. We will also carry out experiments on more subjects to evaluate the generalization performances. Postprocessing approaches will also be designed to remove the recognition errors further. Secondly, the recognition tasks in our study only involved structured terrains in the laboratory environment. In future works, more complicated tasks, including various walking speeds, jogging, and other locomotion modes in daily life, will be investigated. Thirdly, the recognition decisions in our study were discrete in one gait cycle. In future works, we will investigate continuous parameter changes in the locomotion tasks, such as different heights of stairs and different upward locomotion modes (ramps and stairs). The processing algorithm will also be studied to cope with more complicated problems.

\section{Conclusions}

In this study, we designed and preliminarily validated the feasibility of a fuzzy-logic-based algorithm for the locomotion mode and locomotion transition recognition with an active pelvic orthosis. The method purely relied on the inertial signals measured from the thigh, and the sensors were fixed on the exoskeleton. With a proper training process, the fuzzybased algorithm produced comparable recognition accuracies to the existing studies on the same robotic platform. The superiority of the method was that it required no additional sensors on the human body, increasing the convenience in practical applications. The inputs of the fuzzy-logic-based method were the detected peaks and valleys of the pitch angles of the thigh. Combined with the cascaded recognition method, it produced reliable recognition results as long as the detected extreme points were distinguishable between the dynamic locomotion modes. Future works will be focused on onboard training and real-time control of the exoskeleton, investigation of the complicated unstructured terrains, and adaptation to continuous ambulation parameters.

\section{Data Availability}

The data are made available through the corresponding authors' emails.

\section{Conflicts of Interest}

The authors declare no potential conflicts of interest with respect to the research, authorship, and/or publication of this article.

\section{Authors' Contributions}

In this study, Gang Du and Jinchen Zeng analyzed the data and designed the experiments. Jinchen Zeng and Enhao Zheng designed the recognition method. Cheng Gong conducted the experiments. Enhao Zheng guided the writing of the article. Jinchen Zeng and Enhao Zheng wrote the article. Gang Du and Jinchen Zeng contributed equally to this work.

\section{Acknowledgments}

Thanks are due to the China University of Geosciences (Beijing) Information Technology Innovation Experimental Base for the support of this research work. The work is supported by the Undergraduate Education Quality Improvement Project of China University of Geosciences (Beijing) (No. XNFZ202005). 


\section{References}

[1] A. J. Young and D. P. Ferris, "State of the art and future directions for lower limb robotic exoskeletons," IEEE Transactions on Neural Systems and Rehabilitation Engineering, vol. 25, no. 2, pp. 171-182, 2017.

[2] A. B. Zoss, H. Kazerooni, and A. Chu, "Biomechanical design of the Berkeley lower extremity exoskeleton (BLEEX)," IEEE/ASME Transactions on Mechatronics, vol. 11, no. 2, pp. 128-138, 2006.

[3] https://www.cyberdyne.jp/english/products/HAL/.

[4] T. Yan, M. Cempini, C. M. Oddo, and N. Vitiello, "Review of assistive strategies in powered lower-limb orthoses and exoskeletons," Robotics and Autonomous Systems, vol. 64, pp. 120-136, 2015.

[5] D. A. Winter, Ed., Biomechanics and Motor Control of Human Gait: Normal, Elderly and Pathological, University of Waterloo Press, 1991.

[6] C. L. Lewis and D. P. Ferris, "Invariant hip moment pattern while walking with a robotic hip exoskeleton," Journal of Biomechanics, vol. 44, no. 5, pp. 789-793, 2011.

[7] T. Zhang, M. Tran, and H. Huang, "Design and experimental verification of hip exoskeleton with balance capacities for walking assistance," IEEE/ASME Transactions on Mechatronics, vol. 23, no. 1, pp. 274-285, 2018.

[8] F. Giovacchini, F. Vannetti, M. Fantozzi et al., "A light-weight active orthosis for hip movement assistance," Robotics and Autonomous Systems, vol. 73, pp. 123-134, 2015.

[9] K. Junius, M. Degelaen, N. Lefeber, E. Swinnen, B. Vanderborght, and D. Lefeber, "Bilateral, Misalignment-Compensating, FullDOF Hip Exoskeleton: Design and Kinematic Validation," Applied bionics and biomechanics, vol. 2017, 14 pages, 2017.

[10] T. Lenzi, M. C. Carrozza, and S. K. Agrawal, "Powered hip exoskeletons can reduce the user's hip and ankle muscle activations during walking," IEEE Transactions on Neural Systems and Rehabilitation Engineering, vol. 21, no. 6, pp. 938-948, 2013.

[11] K. Seo, J. Lee, Y. Lee, T. Ha, and Y. Shim, "Fully autonomous hip exoskeleton saves metabolic cost of walking," in 2016 IEEE International Conference on Robotics and Automation (ICRA), pp. 4628-4635, Stockholm, Sweden, 2016.

[12] S. K. Banala, S. H. Kim, S. K. Agrawal, and J. P. Scholz, "Robot assisted gait training with active leg exoskeleton (ALEX)," IEEE Transactions on Neural Systems and Rehabilitation Engineering, vol. 17, no. 1, pp. 2-8, 2008.

[13] B. Chen, L. Grazi, F. Lanotte, N. Vitiello, and S. Crea, "A realtime lift detection strategy for a hip exoskeleton," Frontiers in neurorobotics, vol. 12, p. 17, 2018.

[14] A. Parri, K. Yuan, D. Marconi et al., "Real-Time hybrid locomotion mode recognition for lower limb wearable robots," IEEE/ASME Transactions on Mechatronics, vol. 22, no. 6, pp. 2480-2491, 2017.

[15] X. Wu, Y. Ma, X. Yong, C. Wang, Y. He, and N. Li, "Locomotion mode identification and gait phase estimation for exoskeletons during continuous multi-locomotion tasks," IEEE Transactions on Cognitive and Developmental Systems, vol. 13, no. 1, pp. 4556, 2019.

[16] T. Yan, A. Parri, V. Ruiz Garate, M. Cempini, R. Ronsse, and N. Vitiello, "An oscillator-based smooth real-time estimate of gait phase for wearable robotics," Autonomous Robots, vol. 41, no. 3, pp. 759-774, 2017.
[17] E. Zheng, L. Wang, K. Wei, and Q. Wang, "A noncontact capacitive sensing system for recognizing locomotion modes of transtibial amputees," IEEE Transactions on Biomedical Engineering, vol. 61, no. 12, pp. 2911-2920, 2014.

[18] X. Liu and Q. Wang, "Real-time locomotion mode recognition and assistive torque control for unilateral knee exoskeleton on different terrains," IEEE/ASME Transactions on Mechatronics, vol. 25, no. 6, pp. 2722-2732, 2020.

[19] E. Zheng and Q. Wang, "Noncontact capacitive sensing-based locomotion transition recognition for amputees with robotic transtibial prostheses," IEEE Transactions on Neural Systems and Rehabilitation Engineering, vol. 25, no. 2, pp. 161-170, 2016.

[20] C. Gong, D. Xu, Z. Zhou, N. Vitiello, and Q. Wang, "Real-time on-board recognition of locomotion modes for an active pelvis orthosis," in 2018 IEEE-RAS 18th International Conference on Humanoid Robots (Humanoids), pp. 346-350, Beijiing, China, 2018.

[21] F. Gao, G. Liu, F. Liang, and W. H. Liao, "IMU-based locomotion mode identification for transtibial prostheses, orthoses, and exoskeletons," IEEE Transactions on Neural Systems and Rehabilitation Engineering, vol. 28, no. 6, pp. 1334-1343, 2020.

[22] F. Sherratt, A. Plummer, and P. Iravani, "Understanding LSTM network behaviour of IMU-based locomotion mode recognition for applications in prostheses and wearables," Sensors, vol. 21, no. 4, p. 1264, 2021.

[23] C. Gong, D. Xu, Z. Zhou, N. Vitiello, and Q. Wang, "BPNNbased real-time recognition of locomotion modes for an active pelvis orthosis with different assistive strategies," International Journal of Humanoid Robotics, vol. 17, no. 1, article 2050004, 2020. 